\title{
The Role of Superthermal Electrons on The Escaping Ions From The Upper Atmosphere of Titan And Venus
}

\author{
Samy Salem ${ }^{1, *}$, Reda E. Tolba ${ }^{3}$, Waleed M. Moslem ${ }^{1,3}$, and Salah K. El-Labany ${ }^{4}$ \\ ${ }^{1}$ Department of Physics, Faculty of Science, Port Said University, Port Said 42521, Egypt \\ ${ }^{2}$ Basic and Applied Science Department, College of Engineering and Technology, Arab Academy for \\ Science and Technology (AAST), Port Said, Egypt \\ ${ }^{3}$ Centre for Theoretical Physics, The British University in Egypt (BUE), El-Shorouk City 43, Cairo, \\ Egypt \\ ${ }^{4}$ Department of Physics, Faculty of Science, Damietta University, New Damietta, 34517, Egypt \\ * Corresponding author: ssalem92@ hotmail.com
}

\begin{abstract}
The observed escape of ions from the ionosphere of planets and moons is of interest during the last few years. Different mechanisms that can explain the escape of charged particles were assumed. One of the recent mechanisms to explain the ionospheric loss is the plasma expansion approach. In the present work, we investigate the ionic loss from the moon Titan and planet Venus, where their ionospheres suffer from a continues loss reaches to several tons of ions per day. For this purpose, we use a suitable set of hydrodynamic fluid equations to describe the plasma system in either Titan or Venus. Interestingly, it is observed that superthermal electrons are exist in both cases (i.e. Titan and Venus ionosphere). The selfsimilar transformation is used to reduce the basic equations to ordinary differential equations which can be solved numerically to obtain the profiles of the plasma ion density, velocity, and potential during the expansion interval. Furthermore, it is of interest to examine the role of the superthermal electrons on the expansion profile properties.
\end{abstract}

Keywords

Plasma expansion. Titan and Venus ionosphere. Superthermal electrons

\section{INTRODUCTION}

Unlike our planet Earth, Venus and Titan moon lack to a global internally generated magnetic field [1], which make them heavily exposed to the solar wind strikes [2] and [3]. Although Titan is located inside Saturn's magnetic umbrella for most of its orbiting time, it is magnetically unprotected orbiting Saturn for about 7 hours out of Saturn's magnetosphere [4]. Due to the unique atmospheres of Venus and Titan, many attentions have been drawn and also exploration missions have been sent individually to both of them. For example, Venus express (VEX) was sent to Venus and Cassini-Huygens was sent to 
Titan and the whole Saturn's system [5]. According to the Cassini Ion and Neutral Mass Spectrometer measurements of Titan's ionosphere composition, three ions are the major dominant ions in Titan ionosphere [6]. While the observations of Venus' ionosphere indicate that only two ions are the major ions [7].

Titan has the most dens atmosphere of any moon in the solar system, with pressure at the surface larger than the Earth. Thus, Titan has a unique ionosphere, which is usually immersed in Saturn's magnetosphere [8]. Titan ionosphere's has mass loss process and its interaction with the solar wind have been recently reviewed using data from Cassini-Huygens mission [9 and 10]. Indeed, superthermal electron distribution "kappa distribution" has been involved in many space plasma environment such as analyzing and interpreting observational data in Earth's magnetosphere [11], solar wind [12], and Jupiter and Saturn [13]. These works showed that the deviation from isothermal distribution "Maxwellian distribution" is described by superthermal distribution that well-explained the space observation. Richard et al. [14] have shown that Titan's location in Saturn's magnetosphere determines the superthermal electron populations that exist and can precipitate along magnetic field lines into the ionosphere [15]. Furthermore, Szego et al. [16] have shown that the interaction shock between the solar wind and the planetary plasma in Venusian ionosphere could be the origin of the high energetic (superthermal) electrons existing in Venus ionosphere.

Actually $\kappa$ parameter represents the deviation from Maxwellian distribution. In other words, superthermal distribution indicates that there are some electrons have excess of energy, which represents by $\kappa$ parameter. For low values of $\kappa$, it means that the system deviates from Maxwellian case and some electrons acquire excess of energy. While large values of $\kappa$ restore the Maxwellian distribution dominancy again [17 and 18]. Distributions with suprathermal tails have been observed in various space plasmas, where the superthermality parameter $2<\kappa<6$ have been found to fit the observations and satellite data in the solar wind [19 and 20].

Recently, Salem et al. [21] used self-similar plasma expansion approach to solve a plasma model based on the losing phenomenon of Titan atmospheric composition composed of two positive ions with different masses and isothermal electrons. The effects of different plasma parameters, i.e., density and temperature ratios, were studied on the expanding plasma profiles. Later, Moslem et al. [2] investigated the ionic escape from the upper ionosphere of Titan triggered by the solar wind. In this work, a plausible scenario using a hydrodynamic fluid approach for the plasma expansion in the upper ionosphere of Titan, combining (Maxwellian) electrons and three different species of positive ions which interact with the solar wind electrons and protons. It is found that increasing the solar wind proton number density leads to a reduction of the ion escape, while the effect of electrons is opposite stimulating the ion escape. Moreover, the expansion domain does not change for more energetic protons. For a higher temperature contrast between ions and electrons, the depletion rate of the density increases and the ions move faster, leading to a higher ionic loss. Very recently, Salem et al. [3] studied the nature of ionospheric losses from Venus to understand the ionosphere dynamics of this unmagnetized planet. A plausible mechanism that can explain the escape of charged particles involves the solar wind interaction with the upper atmospheric layers of Venus. The behavior of plasma density, velocity, and electric potential, as well as their reliance upon solar wind parameters have been examined. It is found that for noon midnight sites, the oxygen ion-to-electron relative density may be the main factor to enhance the ionic loss. However, the other parameters, like hydrogen density and solar wind density and velocity seem to do not stimulate the runaway ions. For lower dawn-dusk region, the plasma are composed of hydrogen and oxygen ions as well as electrons, but for higher altitudes only hydrogen ions and electrons are encountered. All ionic densities play an important role either to reduce or boost the ionic loss. The streaming solar wind velocity has no effect on the plasma escaping for lower 
altitudes, but it reduces the expansion at higher altitudes. All the above investigations considered a Maxwellian electrons distributions. However, some space observation indicated that some electrons can acquire excess of energy and fellow superthermal distribution [16]. This is the motivation of the present work is to study the effect of superthermal distribution on the ionic loss of Titan and Venus ionosphere.

In this paper, we study the effect of superthermal electron distribution " $\kappa$ - distribution" on the ionospheric composition loss from Venus and Titan upper ionospheres using the plasma expansion approach [21-23]. The paper is arranged as follows: the hydrodynamic fluid equations and the theoretical model are presented in section 2. In section 3, we discuss the numerical results and the summary follows in section 4 .

\section{THEORY AND MODEL EQUATIONS}

We consider unmagnetized, collisionless plasma which is consisting of three positive ions (two positive ions) in case of Titan (Venus), and superthermal electrons. The ions' pressures are assumed to be adiabatic since the particles cool down during the expansion, while the electrons distribution is assumed to be superthermal which is deviated from the Maxwellian distribution with factor $\kappa$. The basic set of fluid equations is governed by the following onedimensional nonlinear partial differential equations [24] as:

For the ions

$$
\begin{gathered}
\frac{\partial n_{j}}{\partial t}+\frac{\partial}{\partial x} n_{j} u_{j}=0 \\
m_{j} n_{j}\left[\frac{\partial}{\partial t}+u_{j} \frac{\partial}{\partial x}\right] u_{j}+e n_{j} \frac{\partial \phi}{\partial x}+\frac{\partial P_{j}}{\partial x}=0 .
\end{gathered}
$$

The superthermal electrons is described by the following distribution [17]

$$
n_{e}=n_{e o}\left[1-\frac{e \phi}{K_{B} T_{e}(\kappa-3 / 2)}\right]^{-\kappa+1 / 2}
$$

Equations (1) - (3) are closed by the plasma neutrality condition as

$$
\sum_{j} n_{j}-n_{e}=0
$$

The quasi-neutrality condition eq. (4) as a plasma approximation is well-known in studies related to plasma at short wave-number or in situations dealing systems having very small Debye length. Therefore, it is obvious that plasma-scale >> Debye length which is enough condition to consider the quasi-neutrality approximation. However, if the plasma-scale $\geq$ Debye length, we have plasma sheath near boundary case that gives rise to the use of Poisson equation. In the present case, it is not possible to obtain a self-similar solution in the presence of Poisson equation. Thus, we use the plasma approximation to investigate the problem and solve the basic equations numerically. It means that under the above conditions, our analysis is satisfactory to explain the phenomena. Furthermore, it will enable us to get a general picture of behavior of the physical system at hand.

The symbols $n_{j}, u_{j}, P_{j}$, and $\phi$ represent the density, velocity, thermal pressure, and electric potential, respectively. The subscript $j$ is 1,2 and 3 in case of Titan stands for first positive ions $\left(\mathrm{HCNH}^{+}\right)$, second positive ions $\left(\mathrm{C}_{2} \mathrm{H}_{5}{ }^{+}\right)$, third positive ions $\left(\mathrm{CH}_{5}{ }^{+}\right)$and e stands for superthermal electrons, respectively. Whereas, the subscript $j$ is 1 and 2 in case of Venus 
stand for the first positive ions $\left(\mathrm{H}^{+}\right)$and second positive ions $\left(\mathrm{O}^{+}\right)$. Here, we use $P_{j}=A n_{j}^{3}$ as the adiabatic fluid pressure where $A$ is some constant and $n_{j}$ is the species ions density. Furthermore, $m_{1}, m_{2}$ and $m_{3}$ are the masses of Titan species $\mathrm{HCNH}^{+}, \mathrm{C}_{2} \mathrm{H}_{5}{ }^{+}$and $\mathrm{CH}_{5}{ }^{+}$ions, respectively. While for Venus $m_{1}$ and $m_{2}$ are the masses of $\mathrm{H}^{+}$and $\mathrm{O}^{+}$ions. To solve Eqs. (1) (4), we introduce the self-similar variable $\xi=x / c_{s} t[25,26]$ to obtain the following normalized ordinary differential equations:

$$
\begin{gathered}
\left(V_{1}-\xi\right) \frac{d N_{1}}{d \xi}+N_{1} \frac{d V_{1}}{d \xi}=0 \\
3 \sigma_{1} N_{1} \frac{d N_{1}}{d \xi}+\left(V_{1}-\xi\right) \frac{d V_{1}}{d \xi}+\frac{d \Phi}{d \xi}=0 \\
\left(V_{2}-\xi\right) \frac{d N_{2}}{d \xi}+N_{2} \frac{d V_{2}}{d \xi}=0 \\
3 Q_{2} \sigma_{2} N_{2} \frac{d N_{2}}{d \xi}+\left(V_{2}-\xi\right) \frac{d V_{2}}{d \xi}+Q_{2} \frac{d \Phi}{d \xi}=0, \\
\left(V_{3}-\xi\right) \frac{d N_{3}}{d \xi}+N_{3} \frac{d V_{3}}{d \xi}=0, \\
3 Q_{3} \sigma_{3} N_{3} \frac{d N_{3}}{d \xi}+\left(V_{3}-\xi\right) \frac{d V_{3}}{d \xi}+Q_{3} \frac{d \Phi}{d \xi}=0 \\
\frac{1}{N_{e}} \frac{d N_{e}}{d \xi}-\frac{\left(\kappa-\frac{1}{2}\right)}{\left(\kappa-\frac{3}{2}-\Phi\right)} \frac{d \Phi}{d \xi}=0 \\
\alpha \frac{d N_{1}}{d \xi}+\beta \frac{d N_{2}}{d \xi}+\gamma \frac{d N_{3}}{d \xi}-\frac{d N_{e}}{d \xi}=0
\end{gathered}
$$

In Eqs. (5) - (12), for Titan, we have $\sigma_{1,2,3}=T_{1,2,3} / T_{e}, Q_{2,3}=m_{1} / m_{2,3}, \alpha=n_{1 o} / n_{e o}$, $\beta=n_{2 o} / n_{e o}$ and $\gamma=n_{3 o} / n_{e o}$. Whereas for Venus, Eqs. (9) and (10) are ignored since we have only two species. Here $n_{1 o}, n_{2 o}, n_{3 o}$, and $n_{e o}$ are the initial densities of the plasma, $n_{j}$ is normalized by the unperturbed density $n_{j o}, u_{j}$ by the ion-acoustic speed $c_{s}=\left(k_{B} T_{e} / m_{1}\right)^{1 / 2}$, $P_{j}$ by $n_{j o} k_{B} T_{j}$ and $\phi$ by $k_{B} T_{e} / e$.

\section{NUMERICAL RESULTS AND DISCUSSION}

We used a suitable numerical code by Mathematica package to solve the basic Eqs. (5) - (12) numerically. To obtain the nontrivial solution, the determinant of the matrix of the independent derivatives must vanish [18], and using the following initial conditions $\mathrm{N}_{1}[0]=0.2, \mathrm{~N}_{2}[0]=0.08, \mathrm{~N}_{3}[0]$ $=0.05, \mathrm{Ne}[0]=1, \mathrm{~V}_{1}[0]=1, \mathrm{~V}_{2}[0]=1, \mathrm{~V}_{3}[0]=1$ and $\Phi[0]=0$. For Venus $\mathrm{N}_{3}$ and $\mathrm{V}_{3}$ are ignored

We have used a typical data from observations for both temperatures and densities and then used them in the relative ratios, for example $\sigma_{\mathrm{j}}=\mathrm{Tj} / \mathrm{Te}_{\mathrm{e}}, \alpha=\mathrm{n} 10 / \mathrm{n}_{\mathrm{e}} 0$. Saturn is far away, at about $10 \mathrm{AU}$ 
from the Sun [27]. At this distance, the solar wind is rarefied with density $0.01-1 \mathrm{~cm}^{-3}$, but still a significant bulk speed e.g., 300-500 km/s, and an average temperature $\sim 10^{4} \mathrm{~K}$ [28]. Among the moons which rotate around Saturn, Titan has no intrinsic magnetic field but its orbit is within Saturn's magnetic field shield for most of the orbital period [29].

In what follows, we analyze the dependence of the profiles of the first positive ion normalized density $\mathrm{N}_{1}$ and velocity $\mathrm{V}_{1}$, as well as electrostatic potential $\Phi$, for both Titan and Venus systems, on the superthermality factor $\kappa$. It is found that the plasma expansion at both of Titan and Venus are affected by the presence of superthermal electrons. The effect of the latter is investigated on positive ion density $\mathrm{N}_{1}$ as depicted in Fig. 1(a) for Titan and in Fig. 1(b) for Venus. It is seen that for higher value of superthermality, the plasma tends to expand more than of lower values. This behavior is the same for Titan and Venus. However, the isothermal electrons (i.e. large $\kappa$ ) have a significant role to make the plasma expansion wider for both Titan and Venus. This could be explained as the superthermal electrons are too fast to make the ions follow them. Thus, the ions cannot go ahead faster to keep track of electrons and the plasma expansion domain decreases. The effect of superthermality on the positive ions velocity $V_{1}$ is shown in Fig. 2(a) for Titan and in Fig. 2(b) for Venus. It is noticed that the ions velocity increases with the increase of $\kappa$, while for the isothermal electrons (i.e. $\kappa=10$ ), they have higher velocity than the non-Maxwellian case. Lastly, the effect of superthermal electrons on the electric potential is shown in the Fig. 3(a) for Titan and in Fig. 3(b) for Venus. It is obviously noticed that the electric potential is affected by the superthermality the same way in both Titan and Venus ionospheres. The value of negativity of the electric potential directly proportional with the superthermality value $\kappa$. Also, the isothermal electrons (higher superthermality) have the most significant effect to increase the electric potential.

\section{CONCLUSION}

This study is dedicated to investigate the effect of superthermal electron population on the escaping composition from the upper ionosphere of Titan and Venus when the magnetic field is ignored. For this purpose, a hydrodynamic system of equations has been solved using the self-similar approach. The numerical calculations showed that both of Venus and Titan are affected by the superthermal electrons. However, Venus is affected by the existence of superhtermal electron with higher rate than of Titan through the enhancement of density depletion, velocity and negative potential. The plasma expansion has been noticed to increase with high deviation from Maxwellian distribution (increasing $\kappa$ ). 


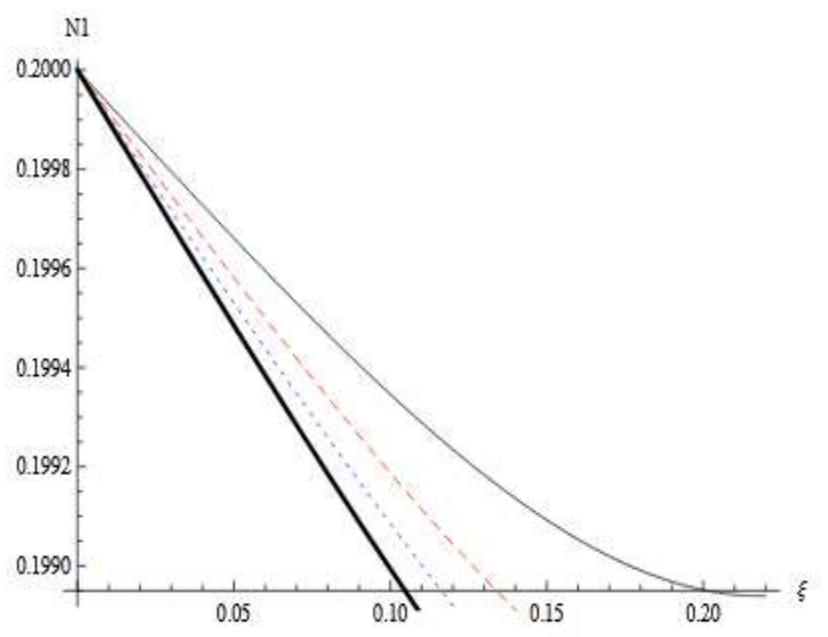

Figure 1(a)

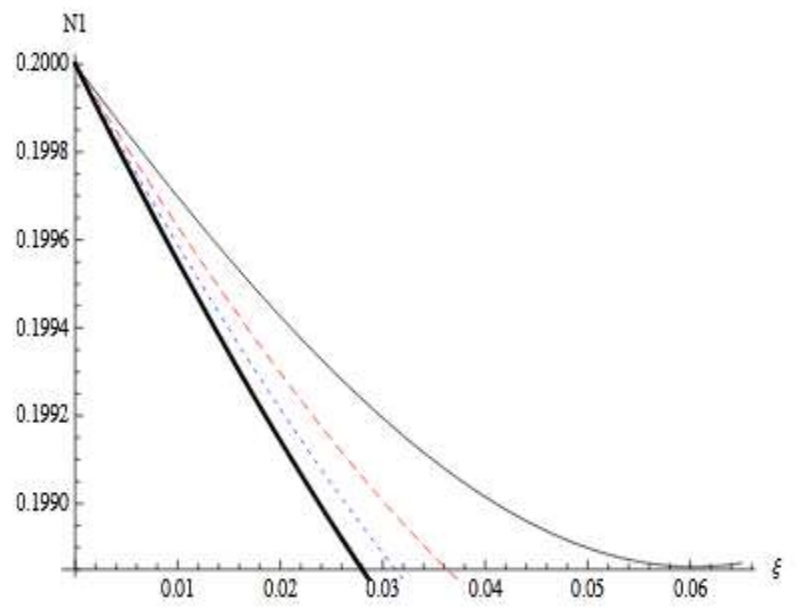

Figure 1(b)

Fig. 1. Normalized positive ions density $\mathrm{N}_{1}$ at (a) Titan and (b) Venus versus self-similar variable $\xi$ for different values of superthermal factor $\kappa$, where $\kappa=3$ (solid line), $\kappa=5$ (dashed line), $\kappa=10$ (dotted line), and for isothermal electron (thick line).

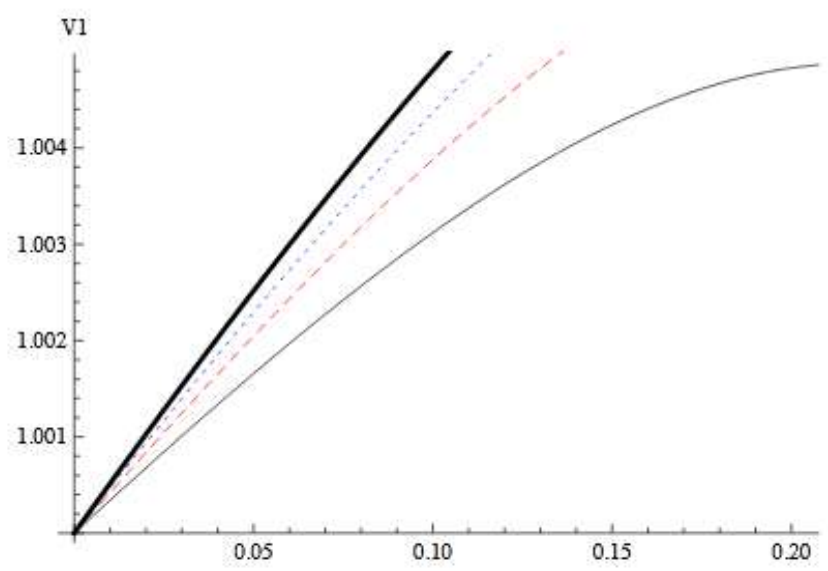

Figure 2(a)

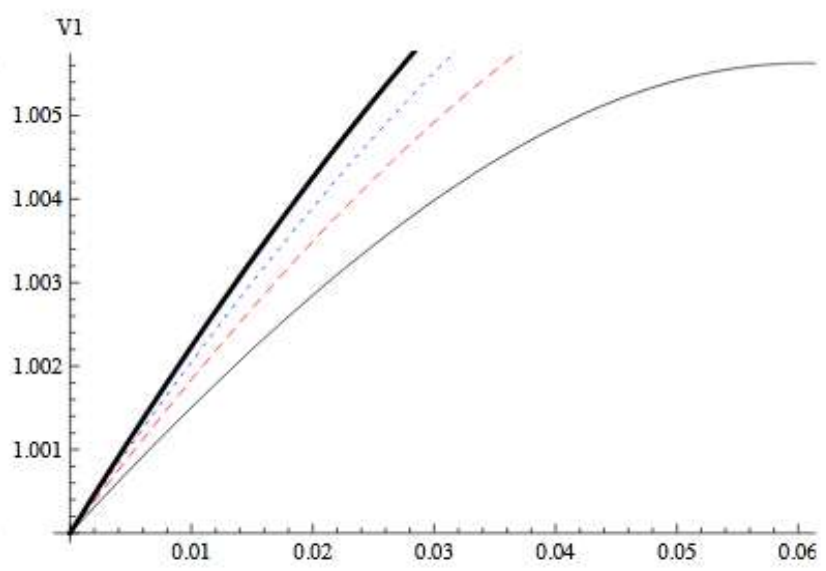

Figure 2(b)

Fig. 2. Normalized positive ions velocity $\mathrm{V}_{1}$ at (a) Titan and (b) Venus versus self-similar variable $\xi$ for different values of superthermal factor $\kappa$, where $\kappa=3$ (solid line), $\kappa=5$ (dashed line), $\kappa=10$ (dotted line), and for isothermal electron (thick line). 


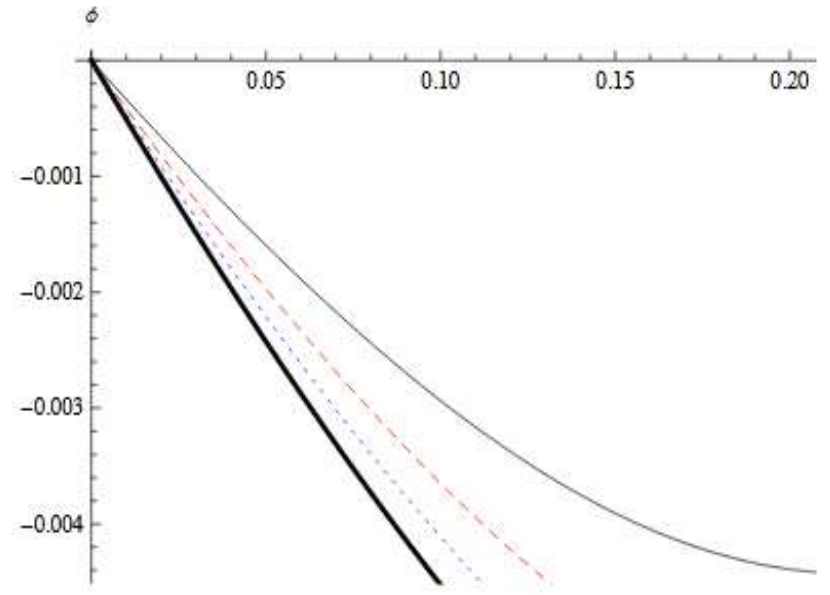

Figure 3(a)

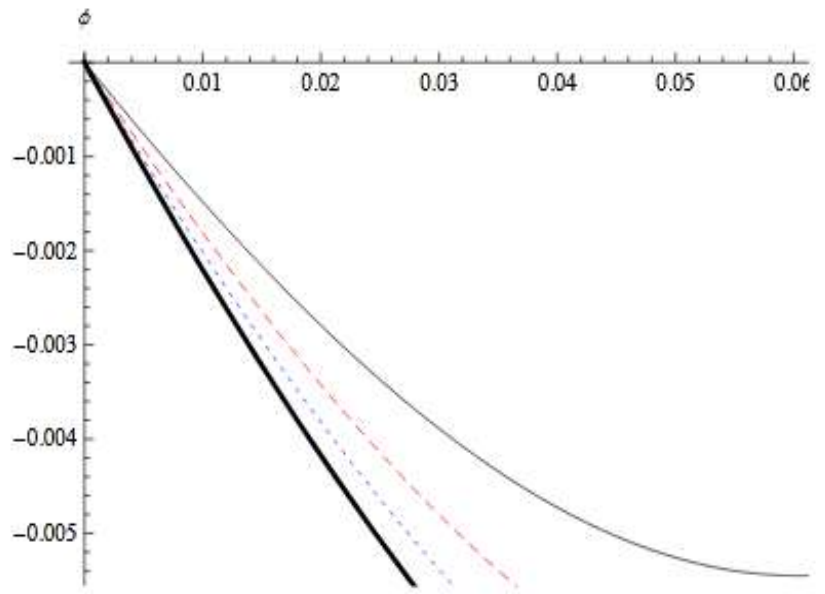

Figure 3(b)

Fig. 3. Normalized electric potential $\Phi$ at (a) Titan and (b) Venus versus self-similar variable $\xi$ for different values of superthermal factor $\kappa$, where $\kappa=3$ (solid line), $\kappa=5$ (dashed line), $\kappa=10$ (dotted line), and for isothermal electron (thick line).

\section{REFERENCES}

[1] SZEGO K., HARRI A., BLANC M., THE PLASMA ENVIRONMENT OF VENUS, MARS AND TITAN: an introduction, Space Science Rev., 162, 1-4, 2011.

[2] MOSLEM W.M., SALEM S., SABRY R., LAZAR M., TOLBA R.E., EL-LABANY S.K., Ion escape from the upper ionosphere of Titan triggered by the Solar wind, Astrophy. Space Sci. 364, 142-149, 2019.

[3] SALEM S., MOSLEM W.M., LAZAR M., SABRY R., TOLBA R.E., AND SCHLIEKEISER R., Ionospheric losses of Venus in the Solar wind, Adv. in Space Res.65, 129-137, 2020.

[4] GARNIER P., WAHLUND J., ROSENQRIST L., MODOLO R., AGREN K., SERGIS N., CAMU P., ANDRE M., GURNETT D., KURTH W., KRIMIGIS S., COATES A., DOUGHERTY M., AND WAITE J., Titan's ionosphere in the magnetosphere, Cassini RPWS results during the T32 flyby, Annales Geophyscae, European Geosciences Unions, 27 (11), pp. 4257-4272, 2009.

[5] FUTAANA Y., CHAUFRAY J., SMITH H., GARNIER P., LICHTENGGER H., DELVA M., GROLLER H., MURA A., Exosphere and Energetic Neutral Atoms of Mars, Venus and Titan, Space Sci. Rev., 162, 213-266, 2011.

[6] CRAVEnS T. E., ROBERTSON I. P., WAITE JR. J. W., ET AL., Composition of Titan's ionosphere, Geophys. Res. Letters, 33, L07105, 2006.

[7] LUNDIN R., Ion acceleration and outflow from Mars and Venus, The plasma Environment of Venus, Mars and Titan, Space Sciences series of ISSI, Springer New York, 37, 309 - 334, 2011.

[8] COATES A.J., WAHLUND J., AGREN K., EDBERG N., CUI J., WELLBROCK A., SZEGO K., Recent results from Titan's Ionosphere, Space Sci. Rev., 162, 85-111, 2011.

[9] CRAVENS T., ROBERTSON I.P., WAITER J.H., YELLE R.V., VUITTON V., COATES A.J., WAHLUND J., AGREN K., RICHARD M., HAYE V., WELLBROCK A., Neubauer F., Model data comparisons for Titan's night side ionosphere, Icarus, 199, 174-188, 2009.

[10] COATES A.J., WELLBROCK A., LEWIS G.R., JONES G.H., YOUNG D.T., CRARY F.J., WAITE J.H., Heavy negative ions in Titan's ionosphere: Altitude and latitude dependence, Planet. Space Sci., 57, 1866-1871, 2009. 
[11] CHRISTON S.P., MITCHELLE D.G., WILLIAMS D.J., FRANK L.A., HUANG C.Y., AND EASTMAN T.E., Energy Spectra of Plasma Sheet ions and electrons from $\sim 50 \mathrm{eV}$ to $\sim 1 \mathrm{MeV}$ during plasma temperature transition, J. Geophys. Res., 93, 2562-2572, 1988.

[12] PIERRARD V. AND LEMAIRE J., Lorenzian ion exosphere model, J. Geophys. Res., 101, 7923-7934, 1996.

[13] KRIMIGIS S.M., CARBARY J.F., KEATH E.P., ARMSTRONG T.P., LANZEROTTI L.J. AND GLOECKLER G., General Characteristics of hot plasma and energetic particles in the Saturnian magnetosphere: Results from the Voyager spacecraft, J. Geophys. Res.: Space Phys., 88, 8871-8872, 1983.

[14] RICHARD M.S., CRAVENS T.E., WYLIE C., WEBB D., CHEDIAK Q., MANDT K., WAITE J.H., RYMER A., BERTUCCI C., WELLBROCK A., WINDSOR A., COATES A.J., An empirical approach to modeling ion production rates in Titan's ionosphere II: Ion production rates on the night side, J. Geophys. Res.: Space Phys., 120, 1281-1298, 2015.

[15] ARRIDGE C.S., ACHILlEOS N., DOUGHERTY M.K., KHURANA K.K., RUSSELL C.T., Modeling the size and shape of Saturn's magnetosphere with variable dynamic pressure, J. Geophys. Res., 111, 227-240, 2006.

[16] SZEGÖ K., DÓBÉ Z., KNUDSEN W. C., NAGY A. F., SHAPIRO V. D., Energetic electrons in the dayside mantle of Venus, J. Geophys. Res., Space Phys., 102, 2175-2183, 1997.

[17] BALUKU T. K., AND HELlBERG M. A., Ion acoustic solitons in a plasma with twotemperature kappa-distributed electrons, Phys. Plasmas 15, 123705 (2008).

[18] PEIRRARD V. AND LAZAR M., KAPPA DISTRIBUTIONS: Theory and Applications in Space plasmas, Solar physics, 267, 153 - 174, 2010.

[19] GLOECKLER G., GEISS J., BALSIGER H., ET AL., The Solar wind ion composition spectrometer, Astron. Astrophys. Supplement series, 92, 267 - 289, 1992.

[20] MAKSIMOVIC M., PIERRARD V., AND RILEY P., Ulysses electron distributions fitter with kappa function, Geophys. Res. Letters, 24, 9, 1151 - 1154, 1997.

[21] SALEM S., MOSLEM W. M., AND RADI A., Expansion of Titan atmosphere, Phys. Plasmas, 24, 901-905, 2017.

[22] MOSLEM W. M., Self-similar expansion of white dwarfs, Astrophys. Space Sci, 342, 351-355, 2012.

[23] ELKAMASH I. S. AND KOURAKIS I., Multispecies plasma expansion into vacuum: the role of secondary ions and superthermal electrons, Phys. Rev. E, 94, 053202, 2016.

[24] CHEN F. F., Introduction to plasma physics and controlled fusion, $3^{\text {rd }}$ ed., Springer International Publishing, Switzerland, 2016.

[25] MOSLEM W. M., EL-SAID A. S., MORSI S. A., YAHIA M. E., SABRY R., EL-LABANY S. K., AND BAHLOULI H., On the formation of nanostructures by inducing confined plasma expansion. Results in Physics, 15, 102696, 2019.

[26] EL-SAID A. S., MOSLEM W. M., AND DJEBLI M., Surface nanostructuring by ion-induced localized plasma expansion in zinc oxide. App Phys Lett 2014;104. 231609.

[27] KALLENRODE MAY-BRITT, Shock waves, Space physics, Springer, Berlin, Heidelberg, 119 $142,1998$.

[28] RICHARDSON J. D., WANG C., AND BURLAGA L. F., The solar wind in the outer heliosphere, Advances in space research, 34, 1, 150 - 156, 2004.

[29] BACKES H., NEUBAUER F. M., DOUGHERTY M. K., ET AL., Titan's magnetic field signature during the first Cassini encounter, Science, 308, 5724, 992 - 995, 2005. 